\title{
Active Matter Clusters at Interfaces
}

\author{
Katherine Copenhagen and Ajay Gopinathan * \\ University of California Merced, Merced, CA, USA
}

Collective and directed motility or swarming is an emergent phenomenon displayed by many self-organized assemblies of active biological matter, such as clusters of embryonic cells during tissue development, cancerous cells during tumor formation and metastasis, colonies of bacteria in a biofilm, or even flocks of birds and schools of fish at the macro-scale. Such clusters typically encounter very heterogeneous environments. What happens when a cluster encounters an interface between two different environments has implications for its function and fate. Here, we study this problem by using a mathematical model of a cluster that treats it as a single cohesive unit that moves in two dimensions by exerting a force/torque per unit area whose magnitude depends on the nature of the local environment. We find that low speed (overdamped) clusters encountering an interface with a moderate difference in properties can lead to refraction or even total internal reflection of the cluster. For large speeds (underdamped), where inertia dominates, the clusters show more complex behaviors crossing the interface multiple times and deviating from the predictable refraction and reflection for the low velocity clusters. We then present an extreme limit of the model in the absence of rotational damping where clusters can

OPEN ACCESS

Edited by: Luca Giomi, Leiden University, Netherlands

Reviewed by: Yaouen Fily, Brandeis University, USA Pragya Srivastava, The Francis Crick Institute, UK

*Correspondence:

Ajay Gopinathan agopinathan@ucmerced.edu

Specialty section: This article was submitted to Biomaterials, a section of the journal Frontiers in Materials

Received: 30 October 2015 Accepted: 15 February 2016

Published: 09 March 2016

Citation:

Copenhagen $K$ and Gopinathan $A$ (2016) Active Matter Clusters at Interfaces.

Front. Mater. 3:13.

doi: 10.3389/fmats.2016.00013 become stuck spiraling along the interface or move in large circular trajectories after leaving the interface. Our results show a wide range of behaviors that occur when collectively moving active biological matter moves across interfaces and these insights can be used to control motion by patterning environments.

Keywords: collective motion, interfaces, environmental heterogeneity, cell clusters, active matter

\section{INTRODUCTION}

Swarming is a widespread biological phenomenon characterized by long range order emerging in a system from local interactions between agents (Sumpter, 2005), such as a swarm of flies (Okubo and Chiang, 1974; Kelley and Ouellette, 2013), or flock of birds (Bialek et al., 2014). Typically, a group of individual organisms self-organize to form a cohesive cluster with directed motility in a spontaneously chosen consensus direction, for example, a school of fish (Herbert-Read et al., 2011), cluster of cells during tumor growth, tissue development and repair (Szabó et al., 2006; Méhes and Vicsek, 2014), or herd of wildebeests (Gueron and Levin, 1993). These types of swarming systems will often encounter a change in the environment, such as a flock of birds flying into a cloud or area of lower air temperature, or a cluster of tumor cells invading different tissue types (Friedl et al., 2012). Single cells have been shown to change their speed and direction when crossing sharp interfaces (Dokukina and Gracheva, 2010). Swarms can also use collective dynamics to find and localize themselves to preferred niches or microenvironments. For example, it has been observed that golden shiners, Notemigonus crysoleucas, which prefer low lighting, will spend more time in dark areas if it is part of a school due to cooperative sensing capabilities of the group (Berdahl et al., 2013). Bacteria have also been shown to take less time to reach a target in the presence of noisy 
chemical concentration gradients in the environment when they are part of a cluster (Shklarsh et al., 2011). Also Escherichia coli clusters modify their own environment by secreting chemicals to create an environmental change between the inside and outside of the cluster in order to trap the E. coli and maintain clustering behaviors (Mittal et al., 2003). It is, therefore, important to understand the effects of spatial environmental changes on a cohesive swarming group. In this paper, we investigate finite swarming clusters moving through heterogeneous environments where agents change their speeds by exerting different forces within each environment. Such changes could arise from the agent's sensing and response to a variety of environmental factors, such as temperature, substrate stiffness, or chemical composition. So, how is the path that a swarm takes affected by the presence of a boundary between different environments, and how does that depend on the properties of the cluster and the environmental change?

Agent based models (Vicsek et al., 1995) and hydrodynamic continuum models (Toner and Tu, 1998) have been used with great success to model natural collective systems (Couzin and Krause, 2003; Sumpter, 2005), and reveal phases and transitions that emerge from the active, far from equilibrium nature of these systems (Couzin et al., 2002; Guillaume and Chate, 2004). Agent based models are implemented by defining a set of interactions and update rules for individuals and then letting the system evolve in time, such systems show phase transitions driven by a wide variety of quantities, including noise, density (Vicsek and Zafeiris, 2012), environmental disorder (Chepizhko et al., 2013), behavioral heterogeneities (McCandlish et al., 2012; Baglietto et al., 2013), and cohesive interaction details (Gazi and Passino, 2004; D'Orsogna et al., 2006). They have also been used to study how swarms use cooperation to achieve specific goals that are useful to biological systems, such as cooperative decision making (Couzin et al., 2005), agent segregation (Belmonte et al., 2008), and obstacle avoidance (Quint and Gopinathan, 2015). Hydrodynamic continuum models, on the other hand, do not treat each agent as an individual, but instead study an average alignment and density profile within the system (Toner et al., 2005). Hydrodynamic swarming models have established active matter as a type of nonequilibrium complex fluid (Toner and Tu, 1995; Marchetti et al., 2013) and provided a unified framework to study phase transitions (Levine et al., 2000; Toner et al., 2005), instabilities (Bertin et al., 2009), and pattern formation (Liu et al., 2013) in active systems.

To answer the question of swarms crossing from one environment to another, we utilize a simplified model for a swarm that assumes a polarized ordered state with velocities correlated across the system (Cavagna et al., 2010) and finite system size, which allows us to examine the overall behaviors of the swarm crossing an interface without necessitating details about individual agent motion as in agent based models, or infinite system sizes as in hydrodynamic models. We consider a swarm as a single cohesive disk-shaped unit, or cluster, each individual agent within the swarm manifests as a force per unit area applied to the disk in the direction of polarization. We then allow the cluster to cross an interface between two differing environments where the portion of the swarm in each environment may apply stronger or weaker forces to the cluster depending on the substrate, resulting in a torque and, therefore, a curved trajectory. We can then map the trajectories of the cluster and measure the resulting relationships between the cluster motion before and after crossing the interface. We find four categories for cluster behavior that depend on two important cluster parameters. The two important parameters are cluster speed, i.e., low speed (overdamped) vs. high speed (underdamped) clusters, and the ratio of the rotational damping to translational friction. It is to be noted that though a prescribed amount of rotational damping arises from the translational friction on the disk, the ratio is a parameter that could be smaller (or bigger) depending on whether the cluster exerts torques to maintain (or resist) rotation.

Our model has predictive capabilities for determining the curved path of a cell cluster at an interface between substrates. The results of which could be extended to suggest possible methods of patterning substrates to direct cell cluster motion. Other regimes of our model provide insight into the behaviors of faster swarms, such as bird flocks and fish schools, moving between heterogeneous environments. Finally, for swarms that exert torques to maintain turning, we show that swarms can display circular paths and trapping at interfaces.

\section{THE MODEL}

We use a mathematical model for a swarming cluster (Figure 1) that treats it as a single cohesive unit that moves on a two dimensional substrate by exerting a force per unit area in some cluster polarization direction (at an angle $\Phi$ with respect to the positive $x$-axis). We then examine a single cluster moving across an interface between two different substrates where the area of the cluster contained on substrate 1 (shown in light green above the solid horizontal line in Figure 1) exerts a force per unit area of $f_{1}$ and the portion on substrate 2 (shown by the dark green area in Figure 1) exerts a force per unit area of $f_{2}$. The force applied on the cluster by every portion of the cluster is in the direction of polarization with a magnitude that depends on the areas

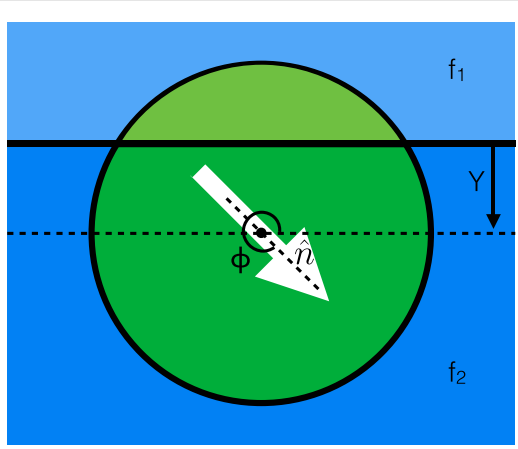

FIGURE 1 | View from above showing the two regions: one above (light green) and the other below (dark green), the interface between two different substrates shown in light and dark blue, respectively. Each region of the cluster propels itself along the polarization direction $(\hat{n}$ at an angle of $\Phi$ with the positive $x$-axis) with a force per unit area of $f_{1}$ for the top region and $f_{2}$ for the bottom region. The cluster center is at a height $Y$ above the interface (in the diagram shown, $Y$ is negative because the center of the cluster is below the interface). 
within each substrate and the substrate dependent forces. The cluster also experiences a friction-like damping force resisting translational motion. Utilizing these details, we can calculate a force on the cluster at any height $Y$ above a horizontal substrate interface (See Supplementary Material for details).

If the cluster is polarized at an angle that is not normal to the interface, there is an asymmetry of the forces on either side of the interface, which results in a torque on the direction of polarization of the cluster that in turn rotates the direction of the force on the cluster and can result in a curved trajectory or a bend in the path of the cluster as it crosses the interface. This torque can be calculated from the force per unit area of the cluster on each substrate along with the distance from the cluster center. From the derived expressions for force and torque (see Supplementary Material), we can find the non-dimensionalized equations of motion for the cluster shown in equations 1-3, where $v_{1}$ and $v_{2}$ are the equilibrium speeds of the cluster in the top and bottom substrates, respectively, and $C$ is the ratio of the angular damping to the translational friction on the disk. In the non-dimensionalized forms of the equations shown below, the translational/frictional damping constant is incorporated into the equilibrium speeds $v_{1}$ and $v_{2}$.

$$
\begin{aligned}
\frac{d^{2} \Phi}{d T^{2}}= & \frac{4}{3 \pi} \sqrt{1-Y^{2}}\left(1+Y^{2}\right)\left(v_{2}-v_{1}\right) \cos (\Phi)-C \frac{d \Phi}{d T} \\
\frac{d^{2} X}{d T^{2}}= & \left(1 / 2\left(v_{1}+v_{2}\right)+1 / \pi\left(v_{1}-v_{2}\right)(\arcsin (Y)\right. \\
& \left.\left.+Y \sqrt{1-Y^{2}}\right)\right) \cos (\Phi)-\frac{d X}{d T} \\
\frac{d^{2} Y}{d T^{2}}= & \left(1 / 2\left(v_{1}+v_{2}\right)+1 / \pi\left(v_{1}-v_{2}\right)(\arcsin (Y)\right. \\
& \left.\left.+Y \sqrt{1-Y^{2}}\right)\right) \sin (\Phi)-\frac{d Y}{d T}
\end{aligned}
$$

We can then use finite difference methods to solve these equations of motion and examine the system subjected to different substrates and initial conditions. In the model, the unit of length is set by the cluster radius, and the unit of time is set by the time taken for the cluster to accelerate from rest to the fraction $(1-1 / \mathrm{e}) \sim 0.63$ of its equilibrium speed. The active nature of the system allows the angular damping and translational friction to not be equal, implying $C \neq 1$. Physically we can expect $C>1$ for situations when swarms exert torques to resist turning, and $C<1$ for cases where swarms exert torques to maintain or persist in turning as seen in systems, such as the persistent turning walker model for Kuhlia Mugil fish (Gautrais et al., 2009). How, then, does the incident angle and ratio of equilibrium speeds in each substrate affect the transmitted angle of the swarm? What is the effect of exerted torques that promote turning?

\section{RESULTS}

Figure 2 shows the four characteristic trajectories for limits of the two important parameters: low velocities (overdamped, friction dominated behaviors), high velocities (underdamped, inertia dominated behaviors), and clusters with angular damping $(C>0)$. At the end, we consider a special limiting case where $C=0$, relevant/applicable to agents that apply torques to promote turning that exactly cancel out any angular damping, resulting in unique cluster behaviors.

\subsection{Cluster Trajectories}

The overall behaviors of the cluster in the presence of angular damping fall into two different categories: refraction/reflection (Figure 3A), and large sweeping curves (Figure 3B). In both cases, the direction that the cluster actively propels itself in $(\hat{n}$ at angle $\Phi$ ) can only accelerate due to torques experienced while it is in contact with the interface. This means that while the cluster is on a single substrate, the angular speed of $\hat{n}$ can only decrease due to rotational damping.

In the low velocity cases $\left(v_{1}=0.01\right.$ in Figure $\left.\mathbf{3 A}\right)$, friction dominates the cluster motion and the cluster moves in a direction parallel to the active propulsion of the cluster $(\vec{v} \| \hat{n})$ at nearly all times. In this friction dominated limit, the system can refract or reflect off of the interface and only comes into contact with the interface once allowing us to measure the incident and refracted angle and make predictions about the behaviors and interactions of the cluster with an interface.

The high velocity case $\left(v_{1}=5\right.$ in Figure $\left.3 \mathbf{B}\right)$ is characterized by the fact that the cluster's translational inertia dominates the direction of cluster motion $(\vec{v})$, for an extended period of time after crossing the interface. In this high velocity case, as the cluster leaves the interface, it will move in a direction that is not necessarily parallel to the direction of $\hat{n}$, resulting in the apparent angular acceleration away from the interface. However, after some time the system will reach equilibrium where $\vec{v} \| \hat{n}$ and the cluster will move in a straight line in some well defined direction. In this case, the momentum of the cluster carries it away from the interface even if the direction of $\hat{n}$ is such that the cluster should be propelled toward the interface meaning that the cluster can rotate and return to the interface a finite number of times before reaching a stable straight trajectory within a single substrate. This high velocity (inertia dominated) case could model the motion of a bird flock crossing an interface between hotter and colder air where their momentum will continue to carry the flock in one direction and the speed and trajectory will gradually stabilize as the flock adjusts to the new environment, potentially traveling in a different direction from that with which it entered.

\subsection{Refraction and Reflection at an Interface}

In this section, we examine the case where the velocity on each substrate is low enough that friction dominates, and the cluster experiences rotational damping $(C>0)$. The cluster in this regime is representative of slow moving natural swarming systems, including cell clusters, and bacteria swarms. A cluster approaching an interface at an angle will begin to turn when it comes into contact with the interface and when contact with the interface is lost it will travel in a straight line in a new direction. We can then measure the resulting angle and the incident angle (labeled $\theta_{f}$ and $\theta_{0}$, respectively, in Figure 2) and compare them to the velocity ratio on the two substrates. To do this, we plot the ratio of the sines of the refracted and incident angles against the ratio of the equilibrium velocities on each substrate. Figure 4A shows the relationship between these ratios for many 


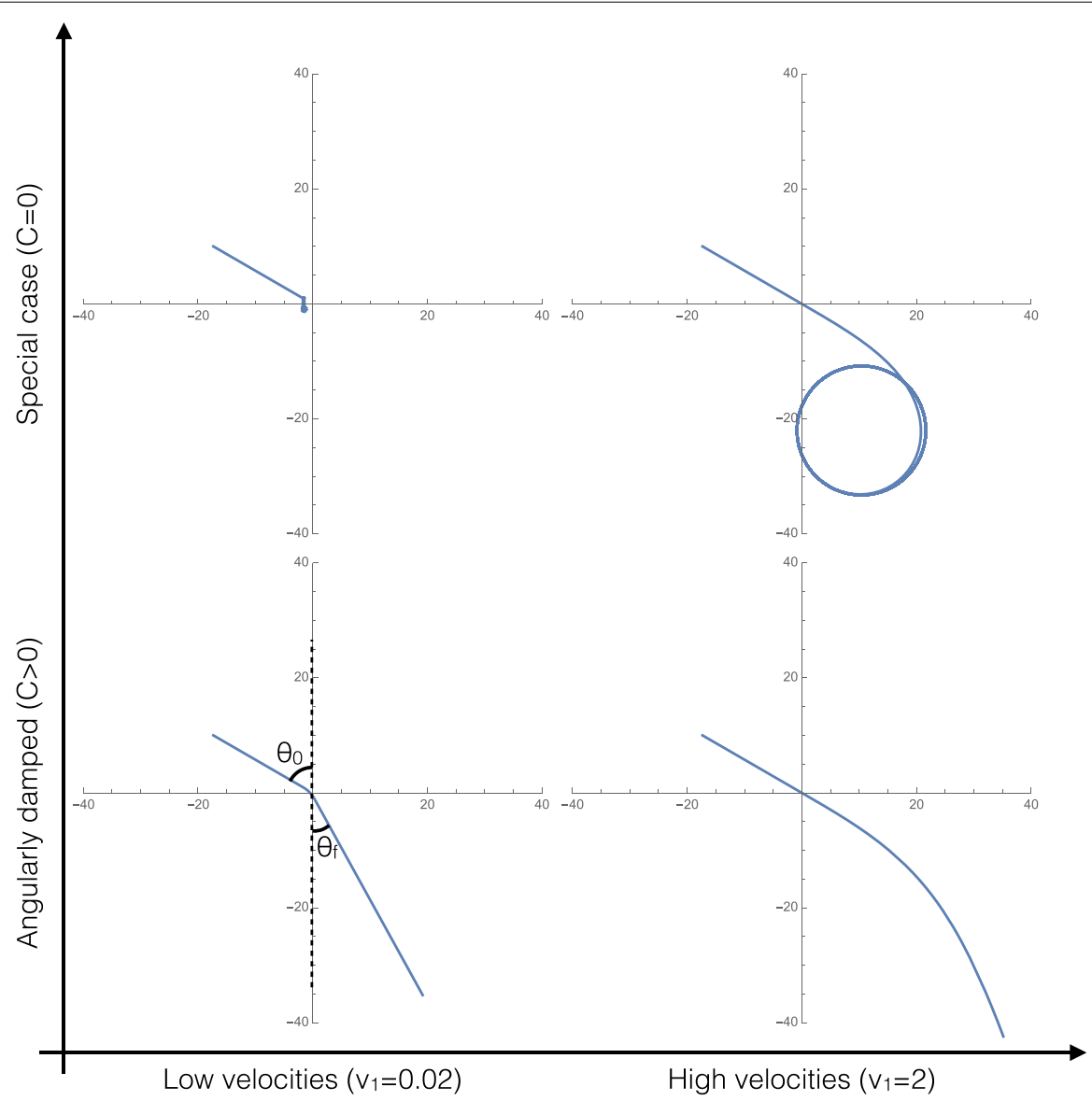

FIGURE 2 | Representative trajectories of the system in each of the four limiting behaviors of the system. In all four cases, $v_{1}=2 v_{2}$.
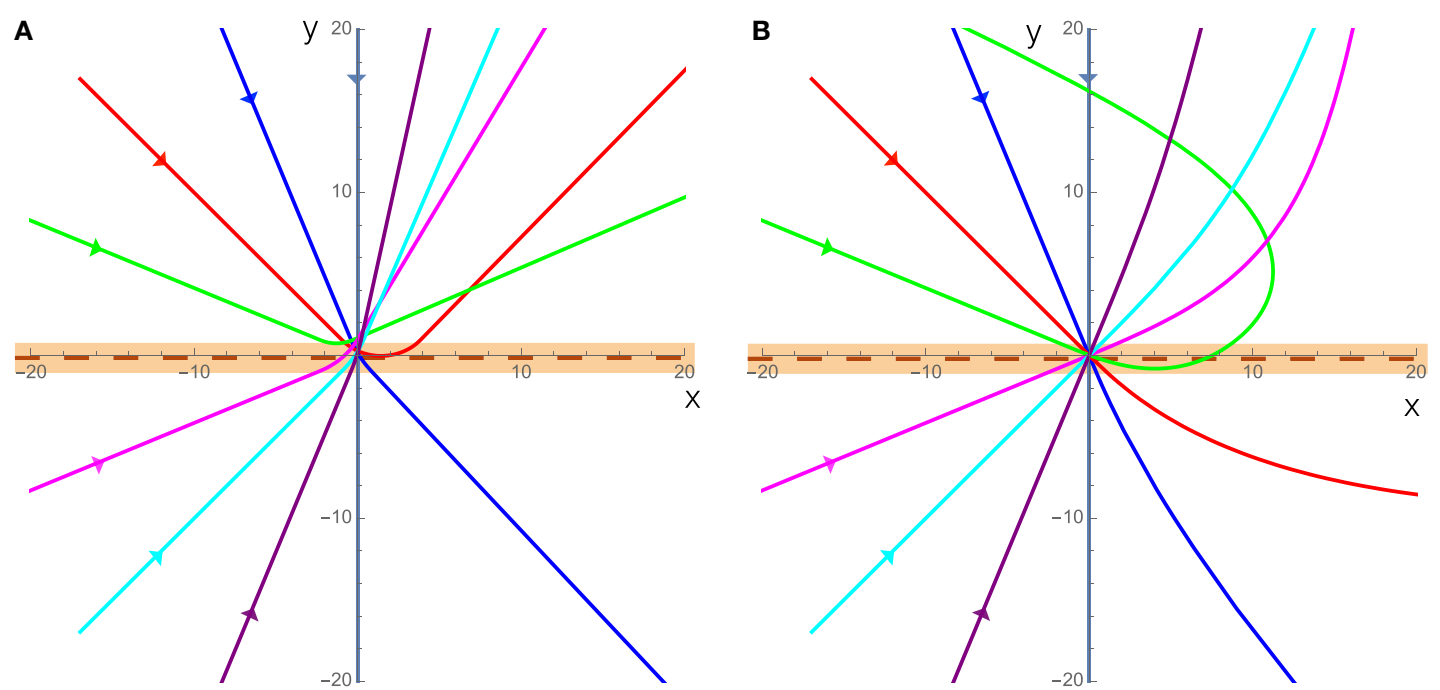

FIGURE 3 | Example trajectories of the cluster, each color is a different incident angle where the incoming cluster is shown by the straight lines entering from the left of each plot $(x<0)$ directed toward the origin, and the clusters are in contact with the interface, which is at $y=0$ (maroon dashed line), when the trajectory is within the orange band. The substrate on the bottom half $(y<0)$ has twice the equilibrium speed as the top substrate $\left(v_{2}=2 v_{1}\right)$ in both cases. (A) For low speeds, where friction dominates cluster motion, the cluster curves while it is in contact with the interface (orange band), and once it leaves the interface it has a well defined straight path along some rotated polarization direction. (B) High velocities, when inertia dominates, result in the cluster starting to curve when it comes into contact with the interface and then large sweeping curves away from the interface as the momentum of the cluster causes it to persist along the previous direction before gradually adjusting to the new substrate. 

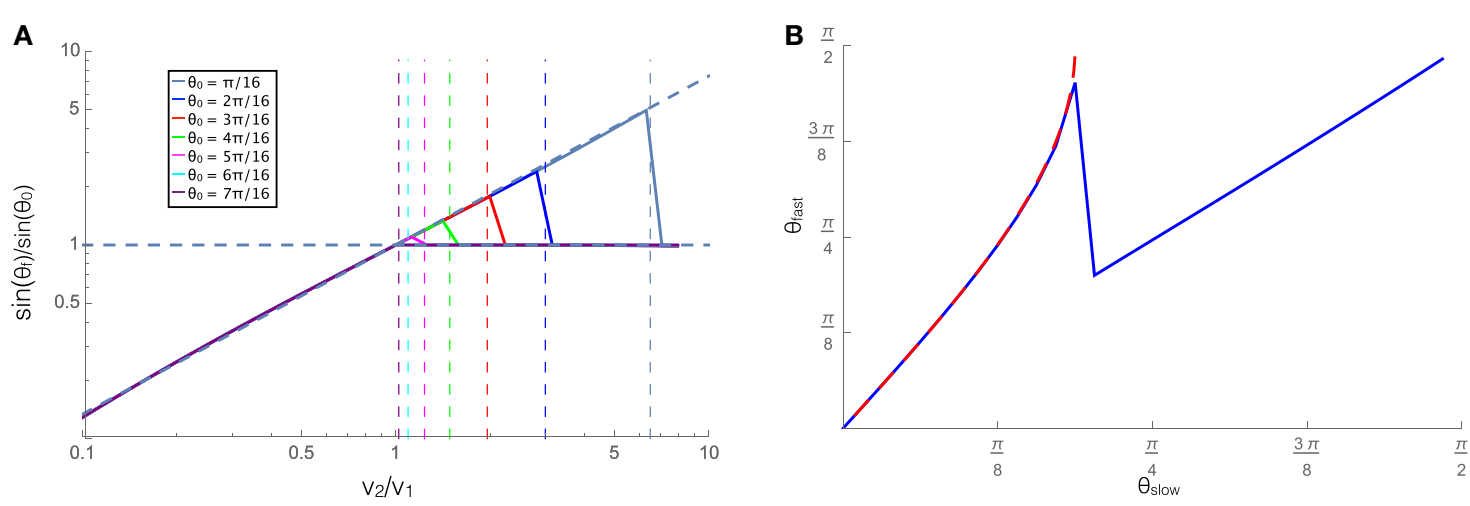

FIGURE 4 | (A) The ratios of the sines of the incident $\left(\theta_{0}\right)$ and refracted $\left(\theta_{f}\right)$ angles plotted against the ratios of the equilibrium speeds in each material, with $v_{1}=0.001$. The vertical lines show the value of velocity ratio where the refracted angle would become $>\pi / 2$, resulting in a reflection, for each initial angle shown in the legend. When the cluster is reflected (right side of the vertical dashed lines), the horizontal line at $\sin \theta_{f} / \sin \theta_{0}=1 \mathrm{shows}$ that the reflected angle is equal to the incident angle. (B) The angle that the trajectory makes (with respect to the normal to the interface) on the faster $\left(\theta_{\text {fast }}\right)$ and slower ( $\left.\theta_{\text {slow }}\right)$ substrates for a cluster moving from the slower substrate to the faster one was shown in blue, and the similar angles for a cluster moving from the faster to the slower substrate were shown in red dashed line. In both these cases, $v_{1}=0.001$ and $v_{2}=0.002$, and $C=1$.

initial angles (see legend), along with a fit line to the numerical data for the refraction angle that leads to the predicted reflections. Applying a similar fit to systems with different values of $C$ leads to the relationship shown in equation 4 . This relation bears a remarkable resemblance to Snell's law for optical paths except with an exponent of $0.87 / C$ instead of unity. Additionally, when moving from a slower substrate to a faster one, the cluster will be reflected off the interface if the transmitted angle should be $\pi / 2$ or greater, i.e., total internal reflection. The velocity ratio where reflection should begin to occur is shown as vertical dashed lines for different incident angles according to the color legend. The predicted reflections align well with the plotted data, where the ratio of the sines for each incident angle begins to reflect at the same point as the vertical dashed lines. The reflection is shown by the horizontal dashed line at 1 , which implies that the reflected angle is equal to the incident angle beyond the velocity ratio that would result in $\theta_{f}>\pi / 2$.

$$
\frac{\sin \theta_{2}}{\sin \theta_{1}}=\left(\frac{v_{2}}{v_{1}}\right)^{0.87 / C}
$$

The trajectories of these clusters are also reversible in time as can be seen in Figure 4B that shows the cluster trajectory angle with respect to normal on the faster substrate plotted against the angle of the trajectory on the slower substrate. The blue solid line shows the case where the cluster is moving from the slower substrate onto the faster one, and drops down to the diagonal line when reflection begins to occur. The red dashed line shows the angles for the faster to slower case. The overlap of these two curves shows that whether moving from faster to slower or vice versa the angles depend only on the substrates and angles and not on the initial substrate of the cluster. The refraction of the cluster into the slower medium while approaching at a large angle from the faster medium is similar to the reported behavior of the golden shiners that collectively turn into darker regions where they move slower (Berdahl et al., 2013).

\subsection{Fast Swarms and Swarms with Exerted Torques to Promote Turning}

In the case where the velocity on each substrate is high enough for inertia to dominate cluster motion, the cluster will still adjust its direction as it passes through the interface but its own momentum will carry it straight across the interface before slowly adjusting its angle depending on the rotations caused by the interface that we can see qualitatively in Figure 3B. This results in the cluster often returning to the interface and interacting with it multiple times making the refracted angle vary from the predictions made for the friction dominated case.

Figure 5 shows the ratio of the incident and refracted angles of clusters versus the ratio of velocities scaled by angular damping similar to Figure 4A. In this plot, the collapse at low velocities for different values of $C$ is shown by the shapes of the markers, $C=0.5$ for the circles, $C=1$ (rotational damping arising from translational friction only) for the triangles and $C=2$ for the squares, the shapes and color legend are consistent across all four plots shown. We can see that at high velocities, the reflected angle varies greatly from the low velocity case where reflected angle was equal to incident angle. This is due to the fact that the cluster passes through the interface before turning around and then passes through the interface again resulting in multiple interactions with the interface and final reflected angles that do not relate in a well defined way to the incident angles. The refracted angle also differs from the low velocity case due to the effects of momentum and inertia at high velocities, the trajectories become complicated and cease to follow a well defined relationship. This behavior could be related to the complex fluid-like motions of starling flocks as they swoop and change directions in large arcing trajectories, possibly due to changing environments from flying over trees or through different altitudes and air temperatures.

Finally, we consider a special case of a swarm that actively promotes rotations by exerting a torque that exactly cancels out any rotational resistance from friction-like damping (resulting in $C=0)$. In this case, two very different behaviors emerge. The first 

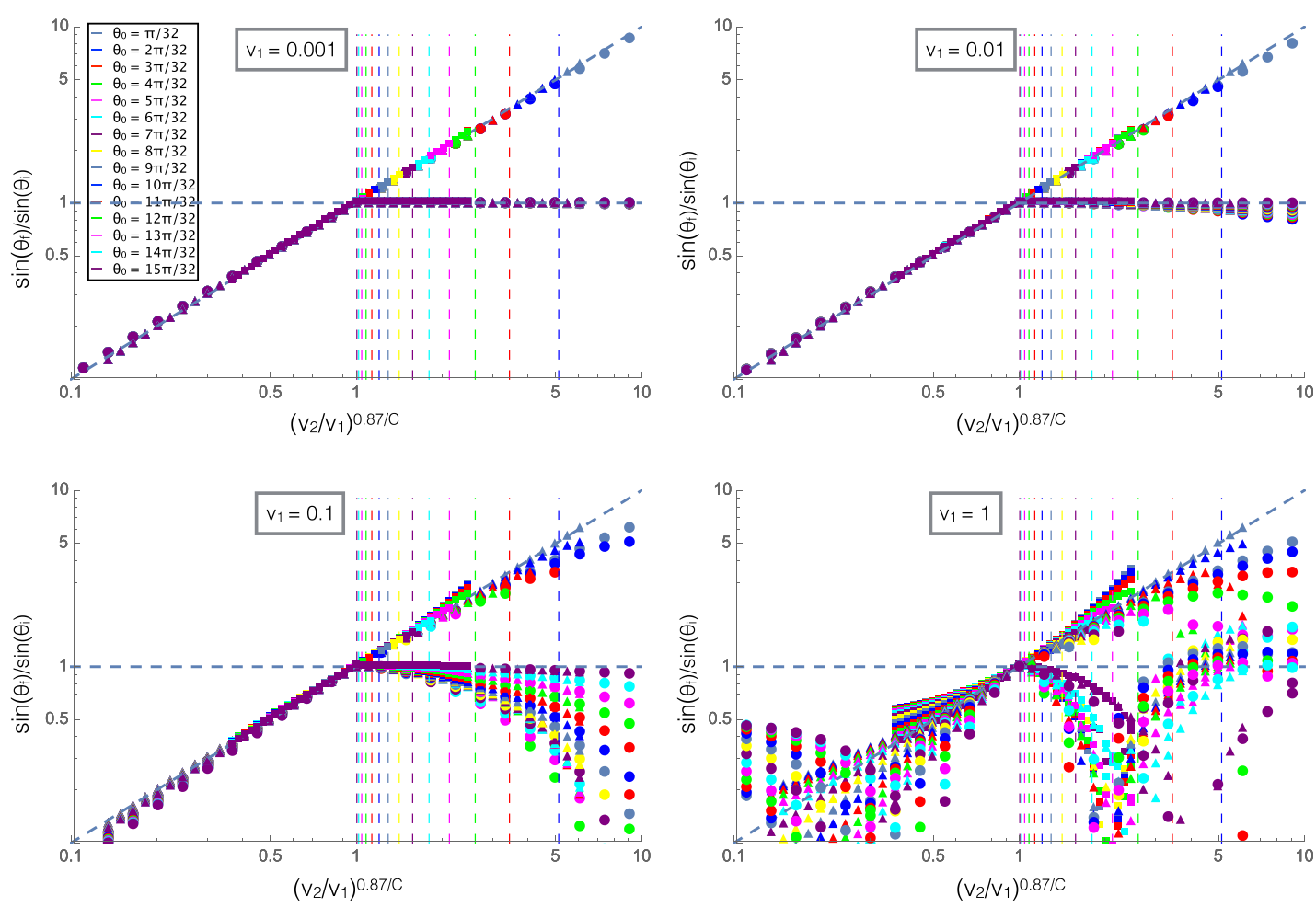

FIGURE 5 | The ratios of the sines of the initial and final trajectory angles measured with respect to the normal to the interface. The velocity on the top substrate $\left(v_{1}\right)$ is labeled for each plot and the color legend for incident angle is consistent across all four plots. Squares are for $C=2$, triangles for $C=1$, and circles for $C=0.5$. The diagonal line is a fit for the low velocity case and the vertical lines are predicted values for the reflections to begin for each incident angle shown by color according to the legend.
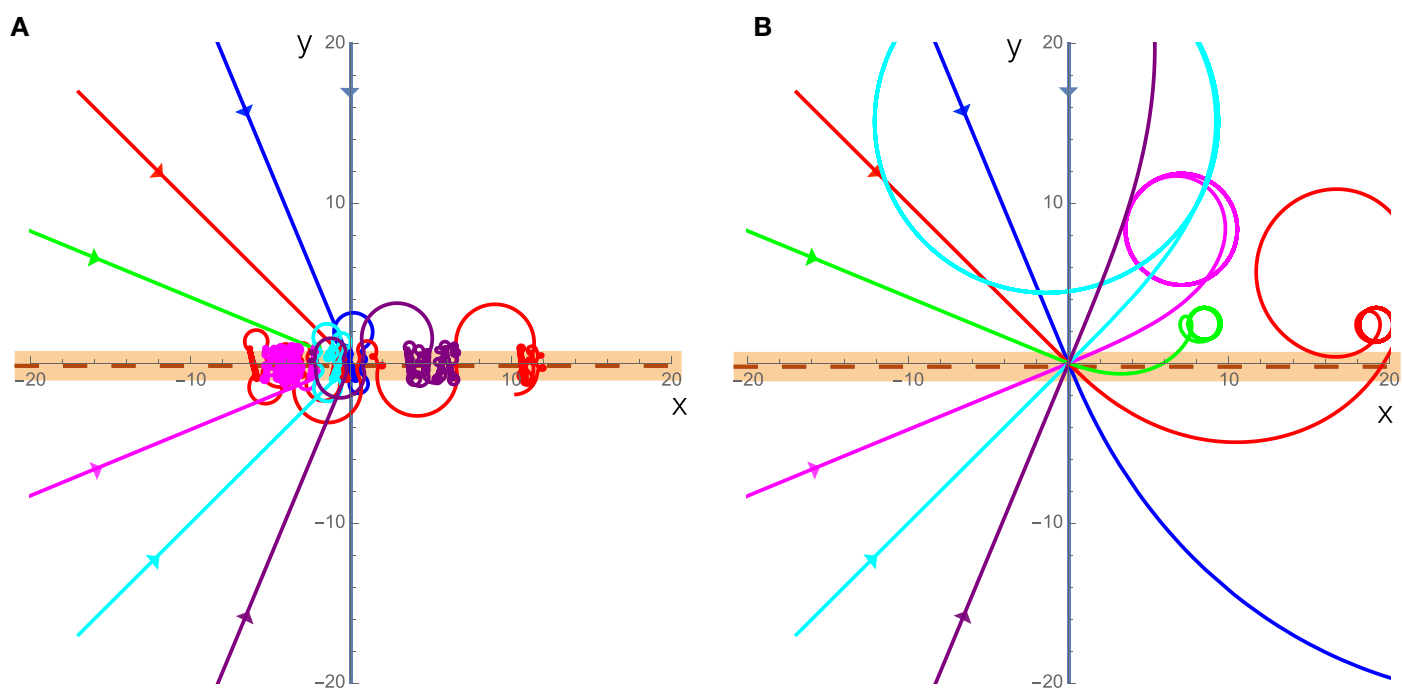

FIGURE 6 | (A) Trajectories for the $C=0$ case with low velocities. $v_{1}=0.001$ and $v_{2}=0.002$, the incident trajectories are the straight lines coming from the left.

(B) Same as (A) but for the high velocity case where $v_{1}=5$ and $v_{2}=10$. The cluster is in contact with the interface while the trajectory is shown in the orange band.

is in the low velocity case where friction dominates the translational motion, though angular momentum is always important due to the fact that $C=0$ independent of velocity. In this case, the cluster will again begin to rotate as it crosses the interface, and experience no angular acceleration once it loses contact with the interface; however, it will continue to rotate along a circular curve and return to the interface some time later. This causes the cluster to become trapped at the interface by always rotating around and returning to the interface without being able to escape as seen in Figure 6A. The second case is for high velocities where the inertia 
of the cluster will carry it past the interface and it may return to the interface or escape but the initial crossing of the interface starts the cluster rotating and the cluster will move in a circle somewhere away from the interface typically on the slower substrate as seen in Figure 6B.

\section{DISCUSSION}

Collective directed motility is a phenomenon that is widespread in biological systems, including cell clusters during tissue development and tumor formation, as well as bacterial biofilms and flocks of birds. In these types of systems, it is reasonable to assume that clusters of collectively moving agents move through changing environments, be it a change in air temperature, stiffness in substrate, or any other change that could result in speed change. We have used a model that treats a swarming cluster as a single cohesive unit with a preferred direction to examine the effects of a swarm moving across an interface between two environments due to a change in speed that occurs in each separate environment.

We found that clusters can display different broad behaviors. The most applicable of which is for slow moving swarms with some angular damping. In this case, a swarming cluster approaching an interface at an angle will undergo some form of refraction or reflection resulting in a new direction that is predictable by a simple relationship between incident and refracted angle and the ratio of the equilibrium speeds on each substrate. Clusters in this regime can also display total internal reflection at the predictable angle where the refraction angle would exceed $\pi / 2$. This regime of our model could represent cell clusters on changing substrates and our predictions could be used to pattern a substrate to direct cluster motion along a desired path.

When the velocities of the cluster are increased on both substrates, the trajectories gradually diverge from the predicted refraction angles and reflections found for low velocities where friction dominates cluster motion. This is due to the inertia of the clusters carrying it quickly across the interface with the inability of the cluster to change directions at a comparable rate. As the cluster velocities continue to increase, the trajectories become sensitive to initial conditions and rotational damping due to the cluster interacting multiple times with the interface or spending less time in contact with the interface than necessary for the cluster to adjust its direction according to the torques present. These kinds of clusters display broad sweeping curved trajectories away from the interface and could provide insight into the impressive collective

\section{REFERENCES}

Baglietto, G., Albano, E. V., and Candia, J. (2013). Gregarious vs individualistic behavior in Vicsek swarms and the onset of first-order phase transitions. Physica A: Statistical Mechanics and its Applications 392, 3240-3247. doi:10.1016/j. physa.2013.03.036

Belmonte, J., Thomas, G., Brunnet, L., de Almeida, R., and Chaté, H. (2008). Self-propelled particle model for cell-sorting phenomena. Phys. Rev. Lett. 100, 248702. doi:10.1103/PhysRevLett.100.248702

Berdahl, A., Torney, C. J., Ioannou, C. C., Faria, J. J., and Couzin, I. D. (2013). Emergent sensing of complex environments by mobile animal groups. Science 339, 574-576. doi:10.1126/science.1225883

Bertin, E., Droz, M., and Grégoire, G. (2009). Hydrodynamic equations for selfpropelled particles: microscopic derivation and stability analysis. J. Phys. A Math. Theor. 42, 445001. doi:10.1088/1751-8113/42/44/445001 motion seen in starling flocks and fast moving fish schools, such as sardines.

In the special case where the cluster experiences no angular damping, possibly due to swarms exerting a torque that counteracts the friction-like resistance that is present in the translational motion, the cluster will either become stuck on the interface for low velocities or stabilize in circular trajectories on a single substrate for high velocities. These types of behaviors may be desirable for certain systems, and our predictions could be used to engineer cell clusters, or robot algorithms to follow desired paths. Trapping a cell cluster on an interface could be useful for separating cells or subjecting them to specific conditions that could then be applied at the interface more easily to cell clusters as individual cells will not display the same kinds of collective modes at an interface. Cell cluster motion has been shown to be controllable by hard boundaries (Doxzen et al., 2013), and our results suggest that a similar strategy could be used with softer substrate interfaces that can be crossed to manipulate cell cluster behaviors.

Our results show possible predictive capabilities for slow moving clusters, such as cells or bacteria moving across changing substrates, as well as possible insight into ongoing questions, such as the behaviors of starling flocks and fish schools as they spiral and curve while they span and cross interfaces between changing environments. Additionally our results suggest possible mechanisms for directing collective systems by way of changing environmental conditions.

\section{AUTHOR CONTRIBUTIONS}

KC and AG designed the project. KC carried out the calculations. $\mathrm{KC}$ and $\mathrm{AG}$ analyzed the results and wrote the paper.

\section{FUNDING}

This work was partially supported by National Science Foundation (NSF) grant EF-1038697 (to AG), a James S. McDonnell Foundation Award (to AG) and an NSF-IGERT graduate fellowship (to KC).

\section{SUPPLEMENTARY MATERIAL}

The Supplementary Material for this article can be found online at http://journal.frontiersin.org/article/10.3389/fmats.2016.00013

Bialek, W., Cavagna, A., Giardina, I., Mora, T., Pohl, O., Silvestri, E., et al. (2014). Social interactions dominate speed control in poising natural flocks near criticality. Proc. Natl. Acad. Sci. U.S.A. 111, 7212-7217. doi:10.1073/pnas. 1324045111

Cavagna, A., Cimarelli, A., Giardina, I., Parisi, G., Santagati, R., Stefanini, F., et al. (2010). Scale-free correlations in starling flocks. Proc. Natl. Acad. Sci. U.S.A. 107, 11865-11870. doi:10.1073/pnas.1005766107

Chepizhko, O., Altmann, E. G., and Peruani, F. (2013). Optimal noise maximizes collective motion in heterogeneous media. Phys. Rev. Lett. 110, 238101. doi:10. 1103/PhysRevLett.110.238101

Couzin, I. D., and Krause, J. (2003). Self-organization and collective behavior in vertebrates. Adv Study Behav. 32, 1-75. doi:10.1016/S0065-3454(03)01001-5

Couzin, I. D., Krause, J., Franks, N. R., and Levin, S. A. (2005). Effective leadership and decision-making in animal groups on the move. Nature 433, 513-516. doi: $10.1038 /$ nature 03236 
Couzin, I. D., Krause, J., James, R., Ruxton, G. D., and Franks, N. R. (2002). Collective memory and spatial sorting in animal groups. J. Theor. Biol. 218, 1-11. doi:10.1006/yjtbi.3065

Dokukina, I. V., and Gracheva, M. E. (2010). A model of fibroblast motility on substrates with different rigidities. Biophys. J. 98, 2794-2803. doi:10.1016/j.bpj. 2010.03.026

D’Orsogna, M., Chuang, Y., Bertozzi, A., and Chayes, L. (2006). Self-propelled particles with soft-core interactions: patterns, stability, and collapse. Phys. Rev. Lett. 96, 104302. doi:10.1103/PhysRevLett.96.104302

Doxzen, K., Vedula, S. R. K., Leong, M. C., Hirata, H., Gov, N. S., Kabla, A. J., et al. (2013). Guidance of collective cell migration by substrate geometry. Integr. Biol. 5, 1026. doi:10.1039/c3ib40054a

Friedl, P., Locker, J., Sahai, E., and Segall, J. E. (2012). Classifying collective cancer cell invasion. Nat. Cell Biol. 14, 777-783. doi:10.1038/ncb2548

Gautrais, J., Jost, C., Soria, M., Campo, A., Motsch, S., Fournier, R., et al. (2009). Analyzing fish movement as a persistent turning walker. J. Math. Biol. 58, 429-445. doi:10.1007/s00285-008-0198-7

Gazi, V., and Passino, K. M. (2004). A class of attractions/repulsion functions for stable swarm aggregations. Int. J. Control 77, 1567-1579. doi:10.1080/ 00207170412331330021

Gueron, S., and Levin, S. A. (1993). Self-organization of front patterns in large wildebeest herds. J. Theor. Biol. 165, 541-552. doi:10.1006/jtbi.1993.1206

Guillaume, G., and Chate, H. (2004). Onset of collective and cohesive motion. Phys. Rev. Lett. 92, 025702. doi:10.1103/PhysRevLett.92.025702

Herbert-Read, J. E., Perna, A., Mann, R. P., Schaerf, T. M., Sumpter, D. J. T., and Ward, A. J. W. (2011). Inferring the rules of interaction of shoaling fish. Proc. Natl. Acad. Sci. U.S.A. 108, 18726-18731. doi:10.1073/pnas.1109355108

Kelley, D. H., and Ouellette, N. T. (2013). Emergent dynamics of laboratory insect swarms. Sci. Rep. 3, 1073. doi:10.1038/srep01073

Levine, H., Rappel, W.-J., and Cohen, I. (2000). Self-organization in systems of selfpropelled particles. Phys. Rev. E 63, 017101. doi:10.1103/PhysRevE.63.017101

Liu, Q.-X., Doelman, A., Rottschäfer, V., de Jager, M., Herman, P. M. J., Rietkerk, M., et al. (2013). Phase separation explains a new class of self-organized spatial patterns in ecological systems. Proc. Natl. Acad. Sci. U.S.A. 110, 11905-11910. doi:10.1073/pnas.1222339110

Marchetti, M. C., Joanny, J. F., Ramaswamy, S., Liverpool, T. B., Prost, J., Madan, R., et al. (2013). Hydrodynamics of soft active matter. Rev. Mod. Phys. 85, 1143. doi:10.1103/RevModPhys.85.1143

McCandlish, S. R., Baskaran, A., and Hagan, M. F. (2012). Spontaneous segregation of self-propelled particles with different motilities. Soft Matter 8, 2527-2534. doi:10.1039/C2SM06960A
Méhes, E., and Vicsek, T. (2014). Collective motion of cells: from experiments to models. Integr. Biol. 6, 831-854. doi:10.1039/C4IB00115J

Mittal, N., Budrene, E. O., Brenner, M. P., and Van Oudenaarden, A. (2003). Motility of Escherichia coli cells in clusters formed by chemotactic aggregation. Proc. Natl. Acad. Sci. U.S.A. 100, 13259-13263. doi:10.1073/pnas.2233626100

Okubo, A., and Chiang, H. C. (1974). An analysis of the kinematics of swarming of Anarete pritchardi Kim (Diptera: Cecidomyiidae). Res. Popul. Ecol. 16, 1-42. doi:10.1007/BF02514077

Quint, D. A., and Gopinathan, A. (2015). Topologically induced swarming phase transition on a 2D percolated lattice. Phys. Biol. 12, 046008. doi:10.1088/1478$3975 / 0 / 0 / 000000$

Shklarsh, A., Ariel, G., Schneidman, E., and Ben-Jacob, E. (2011). Smart swarms of bacteria-inspired agents with performance adaptable interactions. PLoS Comput. Biol. 7:e1002177. doi:10.1371/journal.pcbi.1002177

Sumpter, D. J. T. (2005). The principles of collective animal behaviour. Philos. Trans. R. Soc. B 361, 5-22. doi:10.1098/rstb.2005.1733

Szabó, B., Szöllösi, G. J., Gönci, B., Jurányi, Zs, Selmeczi, D., and Vicsek, T. (2006). Phase transition in the collective migration of tissue cells: experiment and model. Phys. Rev. E74, 061908. doi:10.1103/PhysRevE.74.061908

Toner, J., and Tu, Y. (1995). Long-range order in a two-dimensional dynamical XY model: how birds fly together. Phys. Rev. Lett. 75, 4326-4329. doi:10.1103/ PhysRevLett.75.4326

Toner, J., and Tu, Y. (1998). Flocks, herds, and schools: a quantitative theory of flocking. Phys. Rev. E 58, 4828-4858. doi:10.1103/PhysRevE.58.4828

Toner, J., Tu, Y., and Ramaswamy, S. (2005). Hydrodynamics and phases of flocks. Ann. Phys. 318, 170-244. doi:10.1016/j.aop.2005.04.011

Vicsek, T., Czirók, A., Ben-Jacob, E., Cohen, I., and Shochet, O. (1995). Novel type of phase transition in a system of self-driven particles. Phys. Rev. Lett. 75, 4-7. doi:10.1103/PhysRevLett.75.1226

Vicsek, T., and Zafeiris, A. (2012). Collective motion. Phys. Rep. 517, 71-140. doi:10.1016/j.physrep.2012.03.004

Conflict of Interest Statement: The authors declare that the research was conducted in the absence of any commercial or financial relationships that could be construed as a potential conflict of interest.

Copyright (C) 2016 Copenhagen and Gopinathan. This is an open-access article distributed under the terms of the Creative Commons Attribution License (CC BY). The use, distribution or reproduction in other forums is permitted, provided the original author(s) or licensor are credited and that the original publication in this journal is cited, in accordance with accepted academic practice. No use, distribution or reproduction is permitted which does not comply with these terms. 